\title{
The evolution of innate immune receptors: investigating the diversity, distribution, and phylogeny of immune recognition across eukaryotes
}

\author{
Katherine M. Buckley ${ }^{1}\left[\right.$ [ J Jeffrey A. Yoder ${ }^{2}$ (I) \\ Published online: 15 December 2021 \\ (c) The Author(s), under exclusive licence to Springer-Verlag GmbH Germany, part of Springer Nature 2021
}

Over the past several decades, immunologists have paid considerable attention to characterizing the mechanisms by which jawed vertebrates somatically diversify their antigen receptor repertoires. This effort has resulted in a detailed understanding of adaptive immune systems, particularly in mammals. However, as sequencing technology reveals the genomes and transcriptomes of a broad swath of organisms, we are beginning to appreciate the dynamic evolution of innate immune receptors (IIRs) and their relevance in immune function. IIR provide organisms with the ability to differentiate self (i.e., normal host molecules and cells) from non-self (i.e., microbes or altered host cells), which is key to homeostasis. IIRs can be intracellular, localized on the cell surface, or secreted. Despite their diversity in sequence, ligand specificity, and function within the immune system, many IIRs are characterized by specific protein domains. These common structures are either involved in ligand binding and contain sequence variability in the context of a conserved framework (e.g., immunoglobulin [Ig] domains, leucine-rich repeats [LRR], C-type lectin domains) or mediate downstream signaling responses, and are thus constrained by other cellular systems within the host (e.g., Toll-Interleukin-1 Receptor [TIR], and NACHT domains). Notably, it is well known that many of these domains are present across all eukaryotes, and their functions are not restricted to immune systems. Amazingly, the diversity of IIRs is apparent not only across evolutionary lineages (i.e., among animal or

Katherine M. Buckley

kbuckley@auburn.edu

$\bowtie$ Jeffrey A. Yoder

Jeff_Yoder@ncsu.edu

1 Department of Biological Sciences, Auburn University, Auburn, AL, USA

2 Department of Molecular Biomedical Sciences, Comparative Medicine Institute, Center for Human Health and the Environment, North Carolina State University, Raleigh, NC, USA plant taxa) but is also observed between closely related species. For example, the human IIR repertoire includes toll-like receptors (TLRs), NACHT-like receptors (NLRs), RIG-like receptors (RLR), signal-regulatory proteins (SIRPs), and killer cell immunoglobulin like receptors, (KIRs), as well as MHC molecules mediate that mediate self/non-self recognition. Comparisons of human IIR families to those in nonhuman primates reveal not only significant sequence diversity but also variation in the numbers of IIR orthologs/paralogs present in each species.

Arguably the strongest evolutionary pressure for eukaryotes to maintain genes encoding IIRs is to detect and respond to pathogens. Although the ligands for most IIR are unknown, the well-characterized vertebrate receptors have been shown to recognize molecular features of a myriad of pathogens including viruses, bacteria, fungi, protozoa, and parasitic worms. With logarithmically different life spans, host IIR genes are often subject to diversifying evolutionary pressure to provide an effective defense against rapidly evolving pathogens. This is reflected in the clustered organization of many IIR gene families, which likely promotes gene birth and death events, meiotic recombination, and gene conversion. These diversification strategies can all lead to neofunctionalization, subfunctionalization, and expansion (or contraction) of the recognition repertoire. IIR genes often undergo more rapid and dynamic evolution than most genes encoded in their genomes. In this Special Issue, we explore the diversity of IIRs from plants, vertebrates, invertebrate deuterostomes, a range of protostome invertebrates (i.e., Arthropoda, Mollusca, and Nematoda) and basal metazoans (a cnidarian). Together, this work highlights the great diversity of IIR, and suggests that, to fully understand eukaryotic immune systems, further investigations of these proteins are warranted.

As sessile organisms that lack migratory immune cells, plant immune systems face unique challenges. The evolutionary conflict between plants and plant pathogens is a classic example of "Red Queen" evolution, in which each side 
must constantly evolve to keep pace with the other. Plants express IIR (classically known as pathogen-recognition receptors [PRR]) that detect pathogen-associated molecular patterns (PAMPs) to initiate a first line of defense. In return, plant pathogens secrete virulence factors, known as effectors, which cause disease by suppressing these PAMP-triggered immune (PTI) responses. Consequently, plants encode intracellular nucleotide-binding leucine-rich repeat receptors (NLRs) that recognize effectors and mount effector-triggered immune (ETI) responses, which often results in localized cell death, thereby restricting pathogen growth. In their detailed review, Maruta et al. (2021) focus on two major groups of sensor NLRs in plants that directly or indirectly perceive pathogen effectors and trigger ETI: TIR-NLRs (or TNLs) and helical coiled-coil (CC-NLRs, or CNLs). The authors summarize the structural basis of how TNLs and CNLs recognize effectors, oligomerize, and promote cell death signaling via a network of downstream "helper" NLRs.

In addition to detecting microbes, IIRs (or at least receptors bearing the classical domain structures) play important roles in allorecognition. This is particularly important in colonial marine invertebrate species. Nicotra (2021) reviews our current understanding of allorecognition in the colonial hydroid Hydractinia symbiolongicarpus. As adults, Hydractinia primarily live on marine gastropod shells. In this space-limited environment, when two individuals encounter one another on a single substrate, the colonies undergo either a fusion or rejection. In this context, the evolutionary benefits of fusion are significant: larger colonies not only have greater access to nutrients but can also share metabolic resources and exhibit higher fecundity. However, fusion raises the significant risk of stem cell parasitism; Hydractinia (and other colonial animals) have migratory stem cells that can travel to somatic reproductive structures and differentiate into germ cells. Colonies that have been hijacked in this way can no longer contribute to future generations. This creates an evolutionary imperative for organisms to be able to distinguish their own tissues from those of conspecifics. In Hydractinia, allorecognition is mediated by a single genomic locus that contains at least two allodeterminants (Allorecognition 1 and Allorecognition 2). These genes encode highly polymorphic cell surface proteins that consist of Ig domains and interact through homophilic binding to mediate self/non-self discrimination. This review speculates on homology in the allorecognition systems within all Metazoa.

Innate immune mechanisms have been well characterized in two commonly used Ecdysozoan genetic model systems: Drosophila melanogaster and Caenorhabditis elegans. Arguably, IIR returned to the forefront of immunology with the discovery that Drosophila Toll mediates an antifungal response and that it shared homology with mouse TLRs.
In natural settings, both organisms live in microbe-rich environments and are subject to pathogens. In this issue, Liegeois and Ferrandon (2021) present a comprehensive review of the mechanisms used by Drosophila to detect and respond to microbes. This includes the distinct receptor systems that recognize bacterial, fungal, and viral molecules as well as the different effector arms of host defense. One of the unique aspects of microbial detection in Drosophila is that, rather than binding to microbes directly, many of the IIR recognize host molecules that have been proteolytically cleaved; this mechanism was discovered in the earliest work in which the cleaved molecule spaetzle was shown to interact with Drosophila Toll after fungal infection. While work in model organisms has been invaluable in defining fundamental properties of eukaryotic immune systems that are broadly conserved, it also highlights the distinct characteristics of each organism. In this vein, Pujol et al. (2021) describe the immune system in the nematode $C$. elegans with an eye to aspects that make this system unique. Notably, C. elegans lack migratory, dedicated immune cells and have a somewhat reduced IIR repertoire. Instead of binding to microbes directly, these nematodes initiate immune responses upon detecting disruptions of host tissues, including impaired cellular processes or reduced organelle integrity.

In contrast, Li et al. (2021) present original findings from the lophotrochozoan Biomphalaria glabrata that complement the reviews in this issue based on single-cell transcriptome sequencing of hemocytes (circulating immune cells). Unlike Drosophila and Caenorhabditis, this gastropod snail has a relatively large genome that contains a robust repertoire of IIRs. The authors performed high coverage RNAsequencing on 24 cells: six cells of each of two morphologies (hyalinocytes and granulocytes) from two strains that differ in their response to the parasite Schistosoma mansoni (a resistant strain and a susceptible strain). Analysis of these data reveals that not only are the two strains different at the level of gene expression-resistant snails express more immune genes-but also that the two cell types have distinct functions. Snails partition immune functions such that the granulocytes express IIR (including TLRs and fibrinogenrelated proteins [FREPs]), whereas the hyalinocytes adopt effector functions and express biomphalysins (pore-forming proteins) and thioester-containing proteins. Although this work only begins to characterize the functionality of snail hemocytes, it lays an important foundation for understanding immune responses in this complex, intermediate host.

In contrast to invertebrate species, immune responses in vertebrates are mediated by both an innate and adaptive immunity. A major hallmark of the jawed vertebrate adaptive immune system is the presence of $\mathrm{B}$ and $\mathrm{T}$ lymphocytes that express somatically (V(D)J) recombining Ig genes (that encode antibodies) and T cell receptor (TCR) genes, respectively. (As one might expect, prototypic Ig domains 
were first identified from, and named after, immunoglobulin genes.) Ig domains are present in many IIRs as well as in structural and adhesion proteins. Here, Liberti et al. (2021) investigate the presence of immune-related Ig domaincontaining proteins in invertebrates, including the gastropod FREPs, Down syndrome cellular adhesion molecules (DSCAMs), and variable region-containing chitin binding proteins (VCBPs) of protochordates. The authors discuss the roles that these receptors play, and may play, in gut immunity, particularly within the context of host-microbial interactions. Dornburg and Yoder (2021) also investigate Ig domain-containing proteins but focus instead on membranebound IIRs. Based on the model that the jawed vertebrate adaptive immune system evolved from an ancient receptor encoding a VJ sequence that was separated into $\mathrm{V}$ and $\mathrm{J}$ segments by a RAG-like recombinase (thereby enabling VJ recombination), the authors review extant IIR encoding "joined" (non-recombining) VJ domains. They compare sequences from invertebrates (e.g., Amphioxus), as well as jawless vertebrates (e.g., lamprey and hagfish), and several jawed vertebrate gene families (e.g., SIRPs, and putative remnants of an antigen receptor precursor [PRARPs]), with a specific focus on the novel immune-type receptors (NITRs) in neopterygian fishes.

As fish comprise approximately half of all known vertebrate species and occupy a wide range of ecosystems, the genomically encoded repertoires of immune-related genes provide a wealth of information on how fish immune systems evolved. For example, B30.2 protein domains (also known as PRY/SPRY) are key components of specific subsets of two large families of proteins involved in innate immunity: the tripartite motif proteins (TRIM) and the nucleotide-binding and oligomerization domain (NOD)-like receptors (NLRs), which have experienced lineage-specific expansions in certain fish species. The B30.2 domain bears some structural resemblance to Ig domains, and variability within the B30.2 ligand binding domain has been exploited for pathogen binding and immune functions. In their original article, Suurväli et al. (2021) mine available genome databases from rayfinned fishes to identify gene sequences encoding B30.2 domains and the domain combinations associated with TRIMs, NLRs, and other genes containing B30.2 domains. They tested the impact of taxonomy, genome size, and environmental variables on the size of these gene repertoires, and their findings reveal novel domain structures as well as clade-specific gains and losses, and provide new insights into the origin of these receptor families.

As a group, avian species share many aspects of their immune systems with other vertebrates, but are also characterized by several lineage-specific traits, including substantial loss of immune genes. Birds are hosts for many RNA viruses, including influenza A virus, Newcastle Disease virus, West Nile virus, and coronaviruses. In this special issue, Magor (2021) explores the function and evolution of avian cell surface and intracellular receptors that detect RNA viruses as well as their downstream adaptor proteins. This review provides a detailed report on the genetic and functional roles of the avian TLR and RLR families, and explores the role of the adaptor protein, stimulator of interferon gene (STING), in the RLR signaling pathway leading to immune activation. In contrast, Kaufman (2021) focuses on a different aspect of the avian antiviral immune response: IIR encoded in the MHC. Relative to mammals, chickens have remarkably condensed genomes encoded on several microchromosomes. Microchromosome 16 encodes not only the MHC genes that are associated with adaptive immunity but also many genes involved in innate immunity, including large families of TRIM and B30.2 (and B30.2-like) genes.

Finally, Al-Moussawy et al. (2021) challenge the traditional divide between innate and adaptive immune responses in their review on the current understanding of Paired Immunoglobulin-like Receptors (PIRs). These Ig-domaincontaining receptors in mice are homologous to the human leukocyte immunoglobulin-like receptors (LILR). PIRs are expressed primarily on myelomonocytic cells where they mediate "trained immunity." This phenomenon is characterized by an enhanced activation state in "experienced" monocytes, in which these cells can be more easily activated, perhaps through epigenetic reprogramming. This response has the enhanced secondary response of adaptive immunity, but lacks the antigen specificity and may be important in "finetuning" immune responses. LILRs bind to various ligands that range from classical and non-classical MHC class I (the best-characterized ligands), pathogen-associated proteins, and host immunomodulatory molecules. This recent, exciting work supports a tight cooperation between the innate and adaptive immune systems, and highlights that, in vertebrates, these two systems have been co-evolving to maximize immune responses.

The articles in this special issue highlight the diversity of eukaryotic immune systems from the perspective of IIRs. Altogether, this work not only provides a foundation for understanding the fundamental principles of immune systems that are broadly conserved but also sheds light on the lineage-specific peculiarities that arise in the course of the evolutionary arms race between hosts and microbes. We would like to thank all of the authors for their time and dedication in assembling this fascinating series of articles.

\section{References}

Al-Moussawy M, Abdelsamed HA, Lakkis FG (2021) Immunoglobulinlike receptors and the generation of innate immune memory. Immunogenetics. https://doi.org/10.1007/s00251-021-01240-7 
Dornburg A, Yoder JA (2021) On the relationship between extant innate immune receptors and the evolutionary origins of jawedvertebrate adaptive immunity. Immunogenetics. https://doi.org/ 10.1007/s00251-021-01232-7

Kaufman J (2021) Innate immune genes of the chicken MHC and related regions. Immunogenetics. https://doi.org/10.1007/ s00251-021-01229-2

Li H, Gharamah AA, Hambrook JR, Wu X, Hanington PC (2021) Single cell RNAseq profiling of individual Biomphalaria glabrata immune cells with a focus on immunologically relevant transcripts. Immunogenetics. https://doi.org/10.1007/ s00251-021-01236-3

Liberti A, Natarajan O, Atkinson CGF, Dishaw LJ (2021) Secreted immunoglobulin domain effector molecules of invertebrates and management of gut microbial ecology. Immunogenetics. https:// doi.org/10.1007/s00251-021-01237-2

Liegeois S, Ferrandon D (2021) Sensing microbial infections in the Drosophila melanogaster genetic model organism. Immunogenetics. https://doi.org/10.1007/s00251-021-01239-0
Magor KE (2021) Evolution of RNA Sensing Receptors in Birds Immunogenetics. https://doi.org/10.1007/s00251-021-01238-1

Maruta N, Burdett H, Lim BYJ, Hu X, Desa S, Manik MK, Kobe B (2021) Structural basis of NLR activation and innate immune signalling in plants. Immunogenetics. https://doi.org/10.1007/ s00251-021-01242-5

Nicotra ML (2021) The Hydractinia allorecognition system. Immunogenetics. https://doi.org/10.1007/s00251-021-01233-6

Pujol N, Ewbank JJ (2021) C. elegans: out on an evolutionary limb. Immunogenetics. https://doi.org/10.1007/s00251-021-01231-8

Suurväli J, Garroway CJ, Boudinot P (2021) Recurrent expansions of B30.2-associated immune receptor families in fish. Immunogenetics. https://doi.org/10.1007/s00251-021-01235-4

Publisher's Note Springer Nature remains neutral with regard to jurisdictional claims in published maps and institutional affiliations. 